\title{
DIFFERENTIAL DIAGNOSTICS OF A RADIONECROSIS AND LOCAL TUMORAL RECURRENCE ACCORDING TO ARTERIAL SPIN LABELLING AFTER RADIOSURGERY TREATMENT OF MALIGNANT GLIOMAS OF A BRAIN
}

10.36740/WLek202010110

\author{
Andrey B.Gryazov, Yulia V. Medvedovska, Andrey A. Gryazov \\ INSTITUTE OF NEUROSURGERY OF THE ACADEMICIAN A.P. ROMODANOV OF NATIONAL ACADEMY OF MEDICAL SCIENCES OF UKRAINE, KYIV, UKRAINE
}

\begin{abstract}
The aim: Assessment of a possibility of performing differential diagnostics of the post-beam radio necrosis (PBRN) and the local recurrence (LR) after the carried-out stereotaxic radio surgery (SRS) according to perfusion on the basis of non-contrast pulse arterial backs markings (PASL).

Materials and methods: In our research, we consider results of the stereotactic radiosurgery (SRS) which is carried out to 20 patients with the diagnosis the malignant glioma of a brain (MGB) in respect differential diagnostics of a local tumor recurrence and radionecrosis according to perfusion non-contrast MRT of a research on the basis of the sequence of PASL.

The diagnosis of MGB before carrying out SRS was established to all 20 patients on the basis of a histologic research on data of a biopsy. From them the anaplastic astrocytoma (AnASTs, grade III) and at 14 - a glioblastoma was diagnosed for 6 patients (GBM, grade IV).

Confirmations of the post-beam diagnosis it was carried out on the basis of a histologic research on results of a stereotactic biopsy or a surgical resection.

Traditional methods of medical statistics were applied to processing of the statistical information obtained in a research at all its stages (calculation of average and relative sizes with their errors), also graphic and tabular methods were applied to rational evident statement of the received results.

Results: The diagnosis of a local tumor recurrence according to a relative regional blood-groove on the basis of PASL was exposed to 8 patients and confirmed histologically (to 5 patients underwent surgical resection, 3 - according to biopsy data). Patients with a confirmed diagnosis of relapse according to biopsy were performed in 1 case, repeated radiosurgery and in 2 cases (with a diagnosis of multifocal continued growth of glioblastoma) - irradiation of the entire brain. Radionecrosis was diagnosed in 12 cases. In 6 cases, it was confirmed on the basis of clinical diagnostic data of dynamic observation of patients, in 3 cases - according to surgical resection, and in 3 cases - according to stereotactic biopsy.

According to our data, the average rrCBF based on arterial spin labelling (PASL) rTPmean $\leq 0.8 \mathrm{ml} / 100 \mathrm{~g} /$ minute most likely testified in favor of radionecrosis, average $\geq 1.5 \mathrm{ml} /$ $100 \mathrm{~g} / \mathrm{min}$ - in favor of tumor progression, the maximum rTPmax $\leq 1.3 \mathrm{ml} / 100 \mathrm{~g} / \mathrm{min}$ most likely testified in favor of radio-necrosis, the maximum indicator rTPmax $\geq 1.8 \mathrm{ml} /$ $100 \mathrm{~g} / \mathrm{min}$ - in favor of tumor progression.

Conclusions: According to a relative regional blood flow of rrCBF based on non-contrast PASL perfusion, it is possible to reliably carry out a differential diagnosis of radionecrosis and local tumor recurrence in patients undergoing radiosurgical treatment for malignant gliomas of the brain.
\end{abstract}

KEY WORDS: malignant glioma of the brain, stereotactic radiosurgery, relative cerebral blood flow, pulse arterial spin labelling, radionecrosis, local tumor recurrence

Wiad Lek. 2020;73(10):2160-2164

\section{INTRODUCTION}

Malignant gliomas of the brain are observed in $40 \%$ of patients from the total number of patients with brain tumors $[1,2]$.

The main method for the treatment of gliomas remains surgical resection of tumor tissue, total or subtotal, depending on the location, size and extent of the tumor. In some cases, when the glioma is localized in the brain, in the stem, subcortical, or middlebrain structures of the brain, surgery is associated with a high risk, and in these cases it is advisable to perform a stereotactic biopsy to establish an accurate histological diagnosis, followed by radiotherapy and / or stereotactic radiosurgery $[3,4]$.

Stereotactic radiosurgery can be used either as a single treatment method or in combination with whole brain radiotherapy (WBRT) and chemotherapy $[5,6,7]$.
Postradiation changes after stereotactic radiosurgery (SRS) is often difficult to distinguish from local tumor progression $[8,9]$.

In the case of local recurrence of a tumor after radiosurgical treatment, in the event that surgical resection is not possible, the next stage of treatment can be considered as repeated radiosurgery (single or for several fractions) or irradiation of the entire brain, depending on the size and extent of the process [10].

In case of confirmation of post-radiation changes, further MRI observation and symptomatic therapy are recommended, except in cases where pronounced signs of the mass effect, dislocation syndrome and the clinical condition of the patient require urgent surgical intervention.

Of the non-invasive methods for the differential diagnosis of local tumor recurrence and necrosis, SPECT (sin- 
gle-photon emission computed tomography), MR-spectroscopy and PET are used. In addition to the financial and labor-intensive aspects of these methods, one of their main drawbacks is that relatively low resolution methods may not allow early detection of tumor recurrence, while MRI perfusion methods have higher spatial resolution and demonstrate better results in the differential diagnosis of radionecrosis and recurrence of metastatic lesions $[11,12]$.

Non-contrast MRI perfusion data based on arterial spinal labelling (PASL) can quantitatively and qualitatively determine the density of blood supply and the relative regional blood flow in the tumor focus and in $\mathrm{rCBF}$ and can be used for differential diagnosis of radionecrosis and tumor recurrence, followed by histological confirmation of the diagnosis [13-14]. However, in the literature, there are few data on the use of non-invasive contrast-free MRI perfusion based on arterial spin labelling (PASL) to determine the recurrence of brain metastases after stereotactic radiosurgery [15].

\section{THE AIM}

The aim of our study was to assess the possibility of non-invasive contrastless MRI perfusion based on arterial spin labelling (PASL) in the differential diagnosis of radionecrosis and local tumor recurrence in patients with brain metastases after stereotactic radiosurgery.

\section{MATERIALS AND METHODS}

We carried out a retrospective analysis of perfusion data based on PASL in 20 patients diagnosed with malignant brain glioma, who were treated using the stereotactic radiosurgery in one or more fractions and in which they underwent differential diagnosis of local recurrence (LR) and radionecrosis $(\mathrm{RN})$. Of the total number of patients, there were 13 men and 7 women. The average age of 44 years.

The diagnosis of Malignant Brain Glioma (MBG) before stereotactic radiosurgery was established on the basis of a histological study using stereotactic biopsy data. Anaplastic astrocytoma (AnAST, grade III) was diagnosed in 6 patients and glioblastoma (GBM, grade IV) in 14 patients.

The average tumor volume was $4.0 \mathrm{~cm}^{3}$. The average dose of radiation was $15 \mathrm{Gr}$ (in the range from 12 to 22 $\mathrm{Gr}$ ), depending on the volume and location of the tumor.

After stereotactic radiosurgery, in addition to standard MRI methods, after 1.5 months, 3 and 6 months, the patients underwent non-invasive MRI perfusion based on arterial spin labelling (PASL).

MRI scan was performed on the "Intera" device (Phillips, the Netherlands). The following sequences were used: fast-spin-echo-axial T2WI, spin-echo-axial T1WI, FLAIR, PASL and axial contrast and coronary T1WI.

PASL perfusion of MR images, carried out with the use of multisection sensitive streams with alternative inversion-recovery (FAIR). The FAIR sequence used variable selective and non-selective pulse-inversion (RF) pulses and was performed during TI $1200 \mathrm{~ms}$ between marking and image acquisition.
We selected the T1 range from 800 to $1600 \mathrm{~ms}$ based on the T1 decay of the magnetically-labeled water protons. That is, final FAIR perfusion maps were obtained by subtracting non-selective "inversion-recovery" images from selective images. When conducting the technique of multisection FAIR, we used the image parameters: TR / TE, 2000/15 ms; FOV 24 $\mathrm{cm}$; matrix size $128 \times 128$; NEX 100; profile thickness, $5 \mathrm{~mm}$; section number, 7; and section gap, $2 \mathrm{~mm}$.

To calculate the diagnostic parameters of PASL, we used a qualitative evaluation system based on the intensity of the tumor signal by perfusion. We analyzed the zones of interest and calculated the ratio of the signal intensity with the maximum and average tumor perfusion (rTPmax and rTPmean). MRI data were evaluated, both quantitatively and qualitatively.

Quantitative analysis of the perfusion data was determined by the rCBF values in the brain tumor tissue and the tissue of the normal medulla of the opposite hemisphere of the brain. The measurements were carried out in 6 pixels of the gray and white matter of the contralateral hemisphere. At the same time, measurements in the zone of peritumoral edema, necrosis and hemorrhages were avoided. According to the results of research, $\mathrm{rrCBF}$ compiled PASL cards. The rrCBF values in healthy brain tissue were calculated with respect to the white and gray matter blood flow (WM / GM) and blood flow to metastasis tissue, in relation to the gray matter of the contralateral hemisphere of the brain (MTS / GM). Accurate quantification of the PASL sequence depended on arterial transit time and local tissue and blood relaxation time, regardless of vessel size and oxygenation.

Statistical analysis was performed using IBM SPSS Statistics Version 20 software. Licensed Materials - Property of IBM Corp.

\section{RESULTS AND DISCUSSION}

In total, perfusion data from 20 patients were evaluated according to PASL. In our study, the diagnosis of local tumor recurrence according to the relative regional blood flow based on PASL was confirmed in all 8 cases (Fig. 1).

Radionecrosis was diagnosed in 12 cases and was also confirmed in all cases. An example of clarity can be the case when, according to rrCBF PASL data, rrTPmean and rrTPmax indicators, changes in the glioblastoma focus after stereotactic radiosurgery were considered as a manifestation of radionecrosis, which was subsequently confirmed according to the navigation biopsy data.

CT perfusion data demonstrate multiple hyperperfusion sites that do not distinguish tumor recurrence sites from radionecrosis sites, due to postradiation disorders of the vessel wall permeability, pulse arterial spin labelling (PASL) allows for accurate differentiation of tumor recurrence (white curved arrow), confirmed by biopsy.

Radiation necrosis was subsequently removed surgically, as it behaved volumetrically and caused dislocation of brain structures. According to the histological conclusion in the obtained material, areas of coagulation necrosis were determined, with no "live" tumor tissue in the material (Fig. 2.1. and 2.2.). 


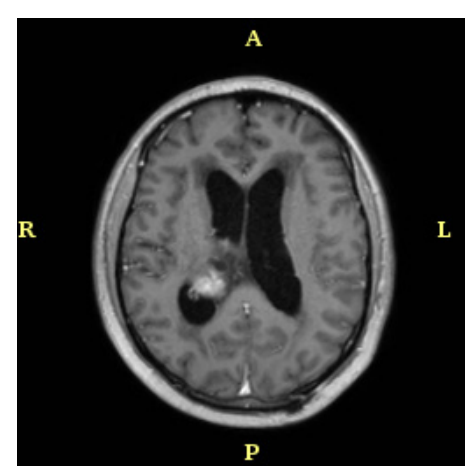

A

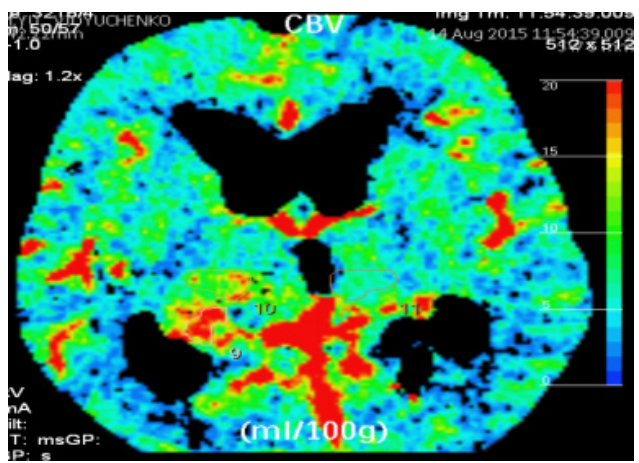

B

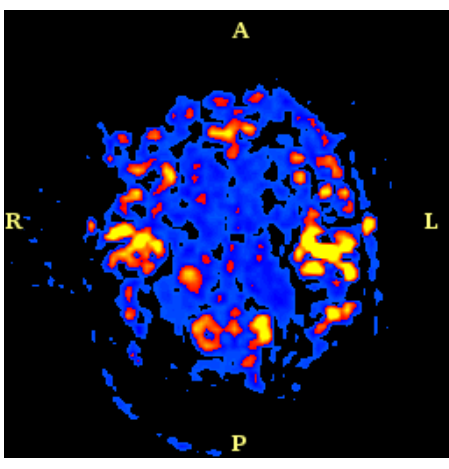

C

Fig.1. Relapse of anaplastic astrocytoma (15 /months after stereotactic radiosurgery). MRI postcontrast T1WI (A), CT perfusion (B), color MRI Perfusion Card (PASL) (C).

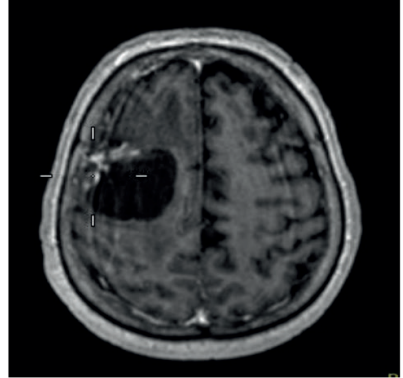

A

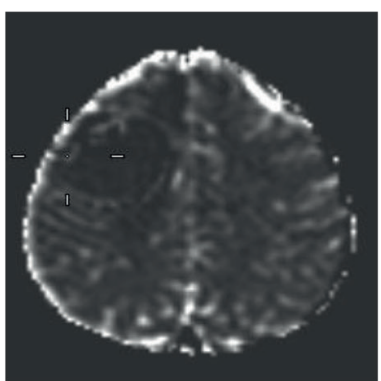

B

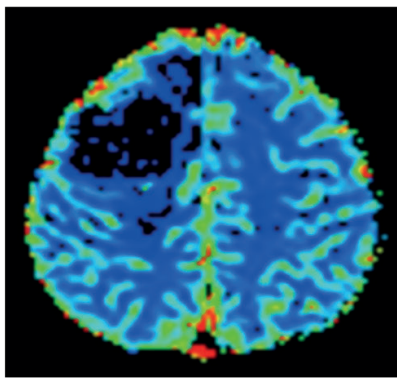

C

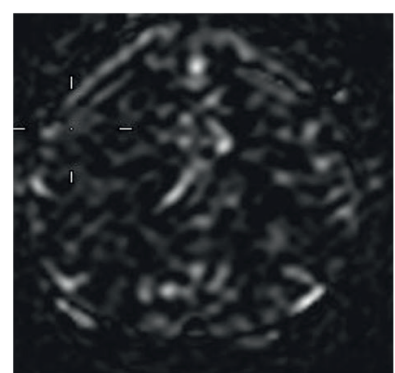

D

Fig. 2.1. A patient with cystic postradiation changes in the right posterior-fronto-parietal region (stereotactic radiosurgery was performed on glioblastoma 24 months ago). MRI postcontrast T1WI (A), black and white and color DSC cards (B and C), PASL cards (D).

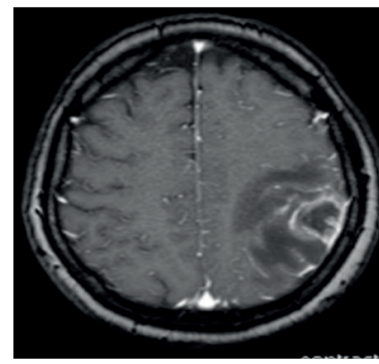

A

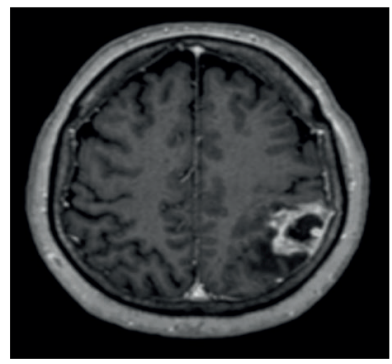

B

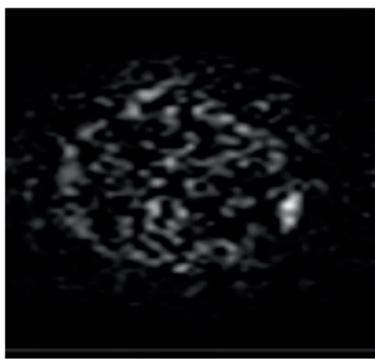

C

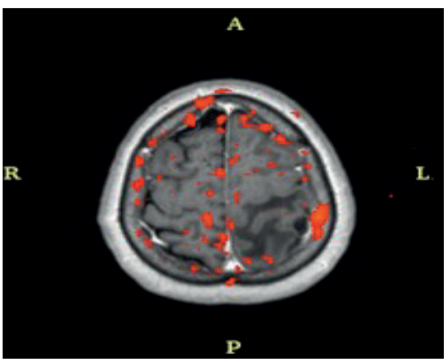

D

Fig.2.2. The patient is 58 years old with suspected continued growth of glioblastoma. (condition after stereotactic radiosurgery). MRI postcontrast T1WI before SRS (A), after 6 weeks (B), rrCBF PASL perfusion after 6 weeks (C), the image with PASL overlay on postcontrast T1WI after 6 months (D).

Using the PASL cards, a suspicious area was found for the presence of "live" tumor tissue with angiogenesis (arrow), however, rTPmax 1.2 and rTPmean values of 0.7 $\mathrm{ml} / 100 \mathrm{~g} / \mathrm{min}$ indicated radionecrosis. According to the PASL maps, a navigation biopsy was performed to obtain biopuncture, then the lesion with the cyst was resected.
The histological conclusion confirmed the presence of a post-radiation focus of coagulation necrosis with a cyst, with no signs of tumor recurrence.

According to MRI postcontrast T1WI before SRS (A) and after 6 weeks (B), the lesion increased in volume by 5 $\mathrm{cm}^{3}$. According to PASL perfusion after 6 weeks (C), rrCBF 
Table 1. Regression Logistic Analysis Result

\begin{tabular}{cccc}
\hline PASL performance & radionecrosis $(\mathbf{n = 2 4 )}$ & relapse $(\mathbf{n = 8})$ & Confidence indicator $\mathbf{9 5 \%}$ \\
\hline rTPmean & $0.8 \mathrm{ml} / 100 \mathrm{gr} / \mathrm{min}$ & $1.5 \mathrm{ml} / 100 \mathrm{gr} / \mathrm{min}$ & $0.002(-3.96-1.01)$ \\
\hline rTPmax & $1.3 \mathrm{ml} / 100 \mathrm{gr} / \mathrm{min}$ & $1.8 \mathrm{ml} / 100 \mathrm{gr} / \mathrm{min}$ & $<0,001(-2.36-0.84)$ \\
\hline
\end{tabular}

Table 2. Indicators of sensitivity, specificity and accuracy according to rrCBF PASL

\begin{tabular}{cccccc}
\hline Indicators PASL & $\mathbf{X}^{\mathbf{2}^{*}}$ & Credibility & Sensitivity (\%) & Specificity (\%) & Accuracy (\%) \\
\hline rrCBF map & 10.329 & $<0.001$ & 86.8 & 100 & 96.6 \\
\hline rrCBF MTS/GM & 6.528 & $<0.001$ & 83.3 & 100 & 94.2 \\
\hline rCBVmean MTS/GM $<0.8$ & 8.764 & 0.002 & 85.5 & 100 & 100 \\
\hline rCBVmean MTS/GM $>1.5$ & 19.538 & $<0.001$ & 96.6 & 96.2 & 98.4 \\
\hline
\end{tabular}

values increased to 1.64 (compared to baseline $1.35 \mathrm{ml} /$ $100 \mathrm{~g} / \mathrm{min}$ to stereotactic radiosurgery) and after 6 months to $1.85 \mathrm{ml} / 100 \mathrm{~g} / \mathrm{min}$., the image with PASL overlay on T1WI (D). According to rrCBF PASL, there is a progression of the disease in the area of brain lining. Surgical resection was performed. The diagnosis of relapse is confirmed on the basis of histological examination.

In general, according to our data, the average $\mathrm{rrCBF}$ based on arterial spin labelling (PASL) rTPmean $\leq 0.8 \mathrm{ml} /$ $100 \mathrm{~g} / \mathrm{min}$ most likely was in favor of radionecrosis, the average figure $\geq 1.5 \mathrm{ml} / 100 \mathrm{~g} / \mathrm{min}-$ in favor of tumor progression, the maximum $\mathrm{rTPmax} \leq 1.3 \mathrm{ml} / 100 \mathrm{~g} / \mathrm{min}$ most likely testified in favor of radionecrosis, the maximum rTPmax $\geq 1.8 \mathrm{ml} / 100 \mathrm{~g} / \mathrm{min}-$ in favor of tumor progression (Table 1).

When considering subgroups of patients with a histologically confirmed diagnosis or with spontaneous regression of a tumor, our data showed high sensitivity and especially specificity (up to $100 \%$ ), most significant for the progression of the process with $\mathrm{rCBF}$ mean MTS / GM $>1.5 \mathrm{ml} /$ $100 \mathrm{~g} / \mathrm{min}$, with a sensitivity of $96.6 \%$, specificity of $100 \%$ and accuracy of $98.4 \%$ (Table 2).

In our observations, PASL perfusion demonstrated significant and reliable indicators of differentiation of radionecrosis with a rCBF index of $<0.8 \mathrm{mml} / 100 \mathrm{~g} / \mathrm{min}$ and a sensitivity of $85.5 \%$, a specificity of $100 \%$ and an accuracy of $96.2 \%$ (p = 0.002 ), and a local tumor recurrence indicator of $\mathrm{rrCBF}>1.5$ $\mathrm{ml} / 100 \mathrm{~g} / \mathrm{min}$ and sensitivity $96.6 \%, 9$ specificity $100 \%$ and accuracy $98.4 \%(\mathrm{p}=<0.001)$, which is similar to literature data $[10,14,15]$.

\section{CONCLUSIONS}

These non-invasive PASL perfusion techniques that determine the relative regional blood flow of $\mathrm{rCBF}$ are sufficiently informative and accurate in the differential diagnosis of radionecrosis and local tumor recurrence in patients undergoing radiosurgery for malignant gliomas of the brain.

\section{REFERENCES}

1. Louis D.N., Perry A., Reifenberger G., et al. The 2016 World Health Organization Classification of Tumors of the Central Nervous System: a summary. Acta Neuropathol. 2016;131:803-820.
2. Omuro A., DeAngelis L.M. Glioblastoma and other malignant gliomas: a clinical review. JAMA. 2013;310:1842-1850.

3. Suchorska B., Weller M., Tabatabai G. et al. Complete resection of contrast-enhancing tumor volume is associated with improved survival in recurrent glioblastoma-results from the DIRECTOR trial. Neurooncology. 2016;18:549-556.

4. Farace P., Amelio D., Ricciardi G.K. et al. Early MRI changes in glioblastoma in the period between surgery and adjuvant therapy. J Neurooncol. 2013;111:177-185.

5. Pinzi V., Orsi C., Marchetti M. et al. Radiosurgery reirradiation for highgrade glioma recurrence: a retrospective analysis. NeurolSci. 2015;36: 1431-1440.

6. Redmond K.J., Mehta M. Stereotactic radiosurgery for glioblastoma. Cureus. 2015;7:413.

7. Navarria P., Ascolese A.M., Tomatis S. et al. Hypofractionated stereotactic radiation therapy in recurrent high-grade glioma: a new challenge. Cancer Res Treat. 2016;48:37-44.

8. Kirkpatrick J.P., Soltys S.G., Lo S.S. et al. The radiosurgery fractionation quandary: single fraction or hypofractionation? Neurooncology. 2017;19(2):38-49.

9. Chao S.T., Ahluwalia M.S., Barnett G.H. et al. Challenges with the diagnosis and treatment of cerebral radiation necrosis. Int J RadiatOncolBiol Phys. 2013;87:449-457.

10. Mitsuya K., Nakasu Y., Horiguchi S. et al. Perfusion weighted magnetic resonance imaging to distinguish the recurrence of metastatic brain tumors from radiation necrosis after stereotactic radiosurgery. J Neurooncol. 2010;.99:81-88.

11. Jarnum H., Steffensen E.G., Knutsson L. et al. Perfusion MRI of brain tumours: a comparative study of pseudo-continuous arterial spin labelling and dynamic susceptibility contrast imaging. Neuroradiology. 2010;52:307-317.

12. Maleki N., Dai W., Alsop D.C. Optimization of background suppression for arterial spin labeling perfusion imaging.MAGMA. 2012;25:127-133.

13. DaiW., Shankaranarayanan A., Alsop D.C. Volumetric measurement of perfusion and arterial transit delay using Hadamard encoded continuous arterial spin labeling. MagnReson Med. 2013;69: 1014-1022.

14. Jiang J., Zhao L., Zhang Y. et al. Comparative analysis of arterial spin Labeling and dynamic susceptibility contrast perfusion imaging for quantitative perfusion measurements of brain tumors. Int J ClinExpPathol. 2014;7:2790-2799.

15. Haller S., Zaharchuk G., Thomas D.L. et al. Arterial spin labeling perfusion of the brain: Emerging clinical applications. Radiology. 2016; 281:337-356. doi: 10.1148/radiol. 2016150789. 
ORCID and contributionship:

Andrey B. Gryazov: 0000-0003-1785-6705 A, D, C, D, E, F

Yulia W. Medvedovskaya: 0000-0001-5119-0316 ${ }^{\text {A, D, C, D, E, F }}$

Andrey A. Gryazov: 0000-0002-2210-1430 A, D, C, D, E, F

\section{Conflict of interest:}

The Authors declare no conflict of interest.

\section{CORRESPONDING AUTHOR}

Yulia W. Medvedovskaya

Institute Of Neurosurgery Of The Academician

A.P. Romodanov Of Namn Of Ukraine

32 Platon Mayboroda st., 04050 Kyev, Ukraine

tel: 066-100-48-94

e-mail:medvedovsky@ukr.net

Received: 14.10 .2019

Accepted: 28.08 .2020

A - Work concept and design, B - Data collection and analysis, C - Responsibility for statistical analysis,

D-Writing the article, $\mathbf{E}$-Critical review, $\mathbf{F}$ - Final approval of the article 